Article

\title{
Dynamic Horizontal Union Algorithm for Multiple Interval Concept Lattices
}

\author{
Yafeng Yang ${ }^{1}\left(\mathbb{D}, \mathrm{Ru}\right.$ Zhang ${ }^{2}$ and Baoxiang Liu ${ }^{1, *}$ \\ 1 College of Science, North China University of Science and Technology, 21 Bohai Road, \\ Tangshan 063210, China; hblgyyf@foxmail.com \\ 2 Department of mathematics and information sciences, Tangshan Normal University, \\ No. 156 Jianshe North Road, Tangshan 063009,China; zhangru1266@126.com \\ * Correspondence: www1673@163.com
}

Received: 3 January 2019; Accepted: 29 January 2019; Published: 10 February 2019

\begin{abstract}
In the era of big data, the data is updating in real-time. How to prepare the data accurately and efficiently is the key to mining association rules. In view of the above questions, this paper proposes a dynamic horizontal union algorithm of multiple interval concept lattices under the same background of the different attribute set and object set. First, in order to ensure the integrity of the lattice structure, the interval concept lattice incremental generation algorithm was improved, and then interval concept was divided into existing concept, redundancy concept and empty concept. Secondly, combining the characteristics of the interval concept lattice, the concept of consistency of interval concept lattice was defined and it is necessary and sufficient for the horizontal union of the lattice structure. Further, the interval concepts united were discussed, and the principle of horizontal unions was given. Finally, the sequence was scanned by the traversal method. This method increased the efficiency of horizontal union. A case study shows the feasibility and efficiency of the proposed algorithm.
\end{abstract}

Keywords: big data; interval concept lattice; horizontal union; sequence traversal

\section{Introduction}

With the era of big data, the complexity of data processing in time and space have become increasingly demanding. Real-time updating of data requires efficient processing of dynamic data. The concept lattice is a powerful tool for data analysis which was proposed by Professor Wille $\mathrm{R}$ in 1982 [1]. It has completeness and accuracy, and has been widely applied in information retrieval, digital library, knowledge discovery, and so on [2-4]. Domestic and overseas scholars have carried out various research on concept lattices that mainly include a construction algorithm and improvement [5-7], rule mining based on concept lattices [8,9], and the fusion of other theories such as fuzzy theory, predicate logic, and rough set theory.

For different needs, some expanded concept lattices have been produced, such as, the fuzzy concept lattice, weighted concept lattice, constraint concept lattice, quantitative concept lattice, expansion concept lattice, rough concept lattice etc. [10-12]. In particular, the classic concept lattice, fuzzy concept lattice and weighted concept lattices, the extent contains the objects which meet all the attributes in the intent. To find the concepts which have partial attributes, we must scan the concept lattice and combine the concepts. The time cost is large especially for large concept lattices. While, in the rough concept lattice, although the concepts which have partial attributes can be searched, there may be a lot of objects which only have an attribute of the intent, thus the support and degree of confidence of constructing association rules will be greatly reduced. In practical applications, we often care about 
the object set which has a certain number or percent of attributes in intent. Then, some pertinent association rules will be mined through a correlation analysis.

Based on the above questions, the interval concept lattice [13] was put forward in 2012 as a collection of objects which had a certain number or percentage of attributes in the connotation. Its expression of concept is $\left(M^{\alpha}, M^{\beta}, Y\right)$. Classical concept lattices are constructed from all attributes with full connotations of extensions, and its expression of concept is $(X, Y)$. Rough concept lattices are composed of the concepts of a maximum attribute set and minimum attribute set containing connotative attributes respectively by upper approximate extension and lower approximate extension. The conceptual form of rough concept lattices is $(M, N, Y)$. Interval concept lattices degenerate into classical concept lattices or rough concept lattices when the parameters are $\alpha=0, \beta=1$ or $\alpha=1 /|Y|, \beta=1$, separately. As will be readily seen, interval concept lattice is a general form of classical concept lattice and rough concept lattice.

On the other hand, with the dynamic change of data, the structure of an interval concept lattice will also change. The association rules are also updated in real time. For example, every day a huge amount of transaction information is generated. If we build the interval concept lattice from the daily trading information, we can only tap the local association rules [14,15]. It cannot provide timely and accurate decision-making for the decision-makers from the overall supermarket shopping system. Therefore, it is very necessary to carry out research on the uniting of interval concept lattices to realize data aggregation. Therefore, the dynamic uniting of interval concept lattices is of great significance.

At present, the main union algorithms of concept lattices are as follows: Reference [16] arranges sub-concept lattices vertically or horizontally in ascending or descending order according to the connotation or extension of the concept; Reference [17] proposes synonymous concepts and updates all father-child nodes according to the relationship between father-child concepts; Reference [18] gives an ordered outline by discussing the relationship between the same concept lattices with the same object set. Since the interval concept lattice has only been proposed for about two years, research on it is limited to the progressive generation algorithm of lattice structure, dynamic compression algorithm and association rules mining algorithm. There is no relevant literature on the uniting of interval concept lattices. The uniting of interval concept lattices is divided into vertical and horizontal uniting. The principle and algorithm of vertical uniting of lattices [19] are studied preliminarily. In this paper, the algorithm for dynamic horizontal uniting of interval concept lattices generated from multiple databases was studied.

The structure is as follows: Section 2 introduces the basic concepts of interval concept lattices, the incremental generation algorithm of interval concept lattices [20] and the related concepts of formal context uniting [21,22]; Section 3 proposes the basic theorem of interval concept lattices' horizontal uniting. On the basis of improving the incremental generation algorithm of interval concept lattices, the horizontal uniting algorithm of interval concept lattices was designed. In Section 4, an example is given to demonstrate the feasibility and efficiency of the algorithm.

\section{Theoretical and Methodological Basis}

For example, in the supermarket shopping system, the promotional manager often pays more attention to the customers who purchase $\mathrm{k}(\mathrm{k}>1)$ kinds of goods or more and the potential demand of these customers, and then carry out product marketing to get the greatest benefit through minimum promotion. However, in the existing concept lattice structure, this kind of query cannot be operated directly, and some union connections or filtrations must be performed. The time and space costs are too high. In order to address this problem, interval concept lattices are required. 


\subsection{Interval Concept Lattice}

Definition 1. For the formal context $(U, A, R)$ and its rough concept lattice $R L(U, A, R),(M, N, Y)$ is the rough concept. Set an interval $[\alpha, \beta](0 \leq \alpha \leq \beta \leq 1)$, then $\alpha$ upper bound extension $M^{\alpha}$ and $\beta$ lower bound extent $M^{\beta}$ are:

$$
\begin{gathered}
M^{\alpha}=\{x|x \in M,| f(x) \cap Y|/| Y \mid \geq \alpha, 0 \leq \alpha \leq 1\} \\
M^{\beta}=\{x|x \in M,| f(x) \cap Y|/| Y \mid \geq \beta, 0 \leq \alpha \leq \beta \leq 1\}
\end{gathered}
$$

$Y$ is the concept intension and $|Y|$ is the number of elements in $Y$, that is base number, $M^{\alpha}$ refers to the objects which may be covered by $\alpha \times|Y|$ attributes or more in $Y . M^{\beta}$ refers to the objects which may be covered by $\beta \times|Y|$ attributes or more in $Y$.

Definition 2. Suppose $(U, A, R)$ is a formal context and $\left(M^{\alpha}, M^{\beta}, Y\right)$ is an interval concept. $Y$ is the intent. $M^{\alpha}$ is the $\alpha$ upper bound extension and $M^{\beta}$ is the $\beta$ lower bound extension.

Definition 3. Suppose $(U, A, R)$ has two interval concepts, $\left(M_{1}^{\alpha}, M_{1}^{\beta}, Y_{1}\right)$ and $\left(M_{2}^{\alpha}, M_{2}^{\beta}, Y_{2}\right)$. If $\left(M_{1}^{\alpha}, M_{1}^{\beta}, Y_{1}\right)$ and $\left(M_{2}^{\alpha}, M_{2}^{\beta}, Y_{2}\right)$ meet $Y_{1} \subseteq Y_{2},\left|Y_{2}\right|-\left|Y_{1}\right|=1, M_{1}^{\alpha}=M_{2}^{\alpha}$ and $M_{1}^{\beta}=M_{2}^{\beta}$, then $\left(M_{1}^{\alpha}, M_{1}^{\beta}, Y_{1}\right)$ is called the redundant concept.

Definition 4. Suppose $(U, A, R)$ has an interval concept, $\left(M^{\alpha}, M^{\beta}, Y\right)$. If it meets $M^{\alpha}=M^{\beta}=\varnothing$, then $\left(M^{\alpha}, M^{\beta}, Y\right)$ is called the empty concept.

Definition 5. Suppose $(U, A, R)$ has an interval concept, $C=\left(M^{\alpha}, M^{\beta}, Y\right)$. If $C$ is neither the redundant concept nor the empty concept, then $C$ is called the existence concept. $L_{\alpha}^{\beta}(U, A, R)$ is a collection of all the existence concepts.

Definition 6. $\overline{L_{\alpha}^{\beta}(U, A, R)}$ refers to all the $[\alpha, \beta]$ interval concepts, which includes: existence concepts, redundant concepts and empty concepts, that is:

$$
\left(M_{1}{ }^{\alpha}, M_{1}{ }^{\beta}, Y_{1}\right) \leq\left(M_{2}{ }^{\alpha}, M_{2}{ }^{\beta}, Y_{2}\right) \Leftrightarrow Y_{1} \supseteq Y_{2},
$$

Then " $\leq$ " is called the partial order relationship of $\overline{L_{\alpha}^{\beta}(U, A, R)}$.

Definition 7. If all the concepts in $\overline{L_{\alpha}^{\beta}(U, A, R)}$ meet " $\leq$ ", then $L_{\alpha}^{\beta}(U, A, R)$ calls interval concept lattice on the formal context $(U, A, R)$.

Definition 8. In the interval concept lattice $L_{\alpha}^{\beta}(U, A, R)$, if $C=\left(M^{\alpha}, M^{\beta}, Y\right) \in L_{\alpha}^{\beta}(U, A, R)$, then the layers of the Lattice Structure is $|A|+1$ and node $C$ is at Layer $|Y|$. In particular, when $Y=\varnothing, C$ was recorded on the zeroth layer.

\subsection{Incremental Construction Algorithm of Interval Concept Lattice (ICAICL)}

Thought of algorithm [11] is as follows.

(1) Calculate the attribute power set $P(A)$ from formal context and let each element $Y$ of power set be intent. Construct the initial node-set $\mathrm{G}$ according to the intension cardinal number in ascending order. For clarity, suppose each concept is a six-point group:

$$
\left(M^{\alpha}, M^{\beta}, Y,\right. \text { Parent, Children, No), }
$$

where $M^{\alpha}, M^{\beta}$ is null set, and Parent $=$ Children $=$ "NULL". 
(2) Set parameters $\alpha, \beta$ and the node of $Y$ is $G=\left(M^{\alpha}, M^{\beta}, Y\right)$. Traversing the intent $Y_{i}$ of every object, if $Y_{i} \subseteq Y$ and $\left|Y_{i}\right| /|Y| \geq \alpha$, then merge the object into $M^{\alpha}$; if $Y_{i} \subseteq Y$ and $\left|Y_{i}\right| /|Y| \geq \beta$, merge the object into $M^{\beta}$.

(3) Firstly, construct the root and end node, then insert other nodes as new ones into the lattice incrementally to form the lattice structure. After inserting a new one, there are three kinds of nodes in the $L_{\alpha}^{\beta,}$ : New node (the node inserted), invariant and update node.

\section{Dynamic Horizontal Union Algorithm}

\subsection{Basic Principles}

Definition 9. In the two formal context $K_{1}=\left(G_{1}, M_{1}, I_{1}\right)$ and $K_{2}=\left(G_{2}, M_{2}, I_{2}\right)$, if $g I_{1} m \Leftrightarrow g I_{2} m$ for each $g \in G_{1} \cap G_{2}$ and $m \in M_{1} \cap M_{2}$, then $K_{1}$ and $K_{2}$ are consistent [12,13]. Otherwise, $K_{1}$ and $K_{2}$ are inconsistent.

Definition 10. Suppose the formal context $\left(U_{1}, A_{1}, R_{1}\right)$ and $\left(U_{2}, A_{2}, R_{2}\right)$ is consistent and they compose interval concept lattice $L_{\alpha_{1}}^{\beta_{1}}\left(U_{1}, A_{1}, R_{1}\right)$ and $L_{\alpha_{2}}^{\beta_{2}}\left(U_{2}, A_{2}, R_{2}\right)$ respectively. When they meet $\alpha_{1}=\alpha_{2}=\alpha$ and $\beta_{1}=\beta_{2}=\beta$, then $L_{\alpha}^{\beta}\left(U_{1}, A_{1}, R_{1}\right)$ and $L_{\alpha}^{\beta}\left(U_{2}, A_{2}, R_{2}\right)$ call consistently, otherwise they are inconsistent.

Definition 11. If interval concept lattice $L_{\alpha}^{\beta}\left(U_{1}, A_{1}, R_{1}\right)$ and $L_{\alpha}^{\beta}\left(U_{2}, A_{2}, R_{2}\right)$ is consistent and meet $U=$ $U_{1}=U_{2}$, we get $L_{\alpha}^{\beta}(U, A, R)$ when we combine them. $L_{\alpha}^{\beta}(U, A, R)$ call horizontal union of $L_{\alpha}^{\beta}\left(U_{1}, A_{1}, R_{1}\right)$ and $L_{\alpha}^{\beta}\left(U_{2}, A_{2}, R_{2}\right)$. Suppose $C_{1} \in L_{\alpha}^{\beta}\left(U_{1}, A, R_{1}\right), C_{2} \in L_{\alpha}^{\beta}\left(U_{2}, A, R_{2}\right)$, then $C=C_{1} \otimes C_{2}$ is the horizontal union of the interval concept.

The concepts in $L_{\alpha}^{\beta}(U, A, R)$ can be divided into two categories. The first is that the number of conceptual connotative attributes in the original structure equals the number of layers of the concepts, and the second is that the sum of the number of conceptual connotative attributes in the two original structures equals the number of layers of the concepts. The specific combination of the two concepts is as follows:

Theorem 1. Suppose $L_{\alpha}^{\beta}\left(U_{1}, A_{1}, R_{1}\right)$ and $L_{\alpha}^{\beta}\left(U_{2}, A_{2}, R_{2}\right)$ is consistent and meet $A_{1}=A_{2}=A$. At the same time suppose $\left(M_{1}^{\alpha}, M_{1}^{\beta}, Y_{1}\right)$ and $\left(M_{2}^{\alpha}, M_{2}^{\beta}, Y_{2}\right)$ are interval concepts in $\overline{L_{\alpha}^{\beta}\left(U_{1}, A, R_{1}\right)}$ and $\overline{L_{\alpha}^{\beta}\left(U_{2}, A, R_{2}\right)}$ respectively. If they meet $Y=Y_{1}=Y_{2}, M^{\alpha}=M_{1}^{\alpha} \cup M_{2}^{\alpha}$ and $M^{\beta}=M_{1}^{\beta} \cup M_{2}^{\beta}$, then $\left(M^{\alpha}, M^{\beta}, Y\right)$ is the interval concept after vertical union.

Theorem 1 shows that for the first kind of concepts, the extension and intension of upper and lower bounds of the concepts themselves remain unchanged before and after uniting. But the flags of conceptual types are different. When the conceptual labels flag equals 2 and flag equals 3 in the lattice structure, they remain unchanged after uniting; when the conceptual label flag equals 1 in the lattice structure, flags may equal 1 or 2 after uniting.

Proof. Since the formal context corresponding to the primitive structure is contained in the formal context corresponding to the united lattice structure, the ternary ordered pairs of concepts in the primitive structure are completely preserved in the united lattice structure. At the same time, there is no change before and after the uniting of empty concepts and redundant concepts. For the existence concepts, the new concepts generated by uniting may make themselves redundant.

For the second kind of concept, $C=\left(M^{\alpha}, M^{\beta}, Y\right) \in L_{\alpha}^{\beta}(U, A, R), C_{1}=\left(M_{1}^{\alpha}, M_{1}^{\beta}, Y_{1}\right) \in$ $L_{\alpha}^{\beta}\left(U, A_{1}, R_{1}\right), C_{2}=\left(M_{2}^{\alpha}, M_{2}^{\beta}, Y_{2}\right) \in L_{\alpha}^{\beta}\left(U, A_{2}, R_{2}\right)$. When $Y_{1} \cap Y_{2}=\varnothing$, taking the upper boundary extension as an example, the case of horizontal union can be divided into the following two types: See Theorems 2 and 3. 
Theorem 2. When $\left\lceil\left|Y_{1}\right| \alpha\right\rceil+\left\lceil\left|Y_{2}\right| \alpha\right\rceil=\left\lceil\left|Y_{1} \cup Y_{2}\right| \alpha\right\rceil, M^{\alpha}=M_{1}^{\alpha} \cap M_{2}^{\alpha}$, verify whether $x_{1}=M_{1}^{\alpha}-M^{\alpha}$ and $x_{2}=M_{2}^{\alpha}-M^{\alpha}$ are empty, if not empty, then bring them into and verify whether $\frac{\left|f(x) \cap\left(Y_{1} \cup Y_{2}\right)\right|}{|Y|} \geq \alpha$. If $\frac{\left|f(x) \cap\left(Y_{1} \cup Y_{2}\right)\right|}{|Y|} \geq \alpha$, add objects to $M^{\alpha}$, or not. Symbol $\lceil x\rceil$ denotes the smallest integer greater than or equal to $x$.

Proof. Determined by the boundary extension itself $\frac{|f(x) \cap Y|}{|Y|} \geq \alpha$, the object satisfying this formula contains at least B attributes, and $Y_{1} \cap Y_{2}=\varnothing$. $\left\lceil\left|Y_{1}\right| \alpha\right\rceil+\left\lceil\left|Y_{2}\right| \alpha\right\rceil=\left\lceil\left|Y_{1} \cup Y_{2}\right| \alpha\right\rceil$ is equivalent to $M^{\alpha}=M_{1}^{\alpha} \cap M_{2}^{\alpha}$. However, $x_{1}=M_{1}^{\alpha}-M^{\alpha}$ or $x_{2}=M_{2}^{\alpha}-M^{\alpha}$ may still contain objects larger than or equal to $\left(\left|Y_{1} \cup Y_{2}\right| \alpha\right)$, so it needs further verification and cannot be eliminated directly.

Theorem 3. When $\left\lceil\left|Y_{1}\right| \alpha\right\rceil=\left\lceil\left|Y_{1} \cup Y_{2}\right| \alpha\right\rceil, M^{\alpha}=M_{1}^{\alpha}$, verify whether $x_{1}=M_{1}^{\alpha}-M^{\alpha}$ and $x_{2}=M_{2}^{\alpha}-M^{\alpha}$ are empty, if not empty, then bring them into and verify whether $\frac{\left|f(x) \cap\left(Y_{1} \cup Y_{2}\right)\right|}{|Y|} \geq \alpha$. If $\frac{\left|f(x) \cap\left(Y_{1} \cup Y_{2}\right)\right|}{|Y|} \geq \alpha$, adds objects to $M^{\alpha}$, or not. $\left\lceil\left|Y_{2}\right| \alpha\right\rceil=\left\lceil\left|Y_{1} \cup Y_{2}\right| \alpha\right\rceil$.

Theorem 4. Supposes that $L_{\alpha}^{\beta}(U, A, R)$ is the interval concept lattice obtained by the horizontal uniting of $L_{\alpha}^{\beta}\left(U, A_{1}, R_{1}\right)$ and $L_{\alpha}^{\beta}\left(U, A_{2}, R_{2}\right)\left(A_{1} \cap A_{2}=\varnothing\right)$, then the m-level node of $L_{\alpha}^{\beta}(U, A, R)$ is $C_{\left|A_{1}\right|+\left|A_{2}\right|}^{m}=$ $C_{\left|A_{1}\right|}^{m}+C_{\left|A_{1}\right|}^{m-1} C_{\left|A_{2}\right|}^{1}+C_{\left|A_{1}\right|}^{m-2} C_{\left|A_{2}\right|}^{2}+\ldots+C_{\left|A_{1}\right|}^{1} C_{\left|A_{2}\right|}^{m-1}+C_{\left|A_{2}\right|}^{m}$.

Here $C_{\left|A_{1}\right|}^{m}$ refers to all interval concepts whose number of intension attributes is $\mathrm{m}$ in $\overline{L_{\alpha}^{\beta}\left(U, A_{1}, R_{1}\right)}, C_{\left|A_{1}\right|}^{m-1} C_{\left|A_{2}\right|}^{1}$ refers to all interval concepts whose number of intension attributes is $m-1$ in $\overline{L_{\alpha}^{\beta}\left(U, A_{1}, R_{1}\right)}$ and all interval concepts whose number of intension attributes is 1 in $\overline{L_{\alpha}^{\beta}\left(U, A_{2}, R_{2}\right)}$ merge horizontally.

\subsection{Algorithmic Design}

In order to generate interval concept lattices while retaining all interval concepts, including existing concepts, redundant concepts and empty concepts, and effectively improve the uniting efficiency of interval concept lattices, the existing incremental interval concept lattice generation algorithm needs to be modified first, and on this basis, a dynamic horizontal uniting algorithm of multiple interval concept lattices is proposed.

\subsubsection{Improved Progressive Generation Algorithms for Interval Concept Lattices}

In order to distinguish between different interval concepts, concept nodes are defined and stored in structured form as follows:

$$
\text { flag }\left|M^{\alpha}\right| M^{\beta}|Y| \text { parent } \mid \text { children } \mid \text { no }
$$

It is defined in the following form.

struct concept

$\left\{\right.$ string $M_{i}^{\alpha} ; M_{i}^{\alpha} M_{i}^{\beta} ; Y_{i} ;$

struct $Y$, parent, children;

int flag;

The concept of category is marked by flag. When flag $=1$, flag $=2$ and flag $=3$, stored concept is exist concept, redundant concept and empty concept separately. 


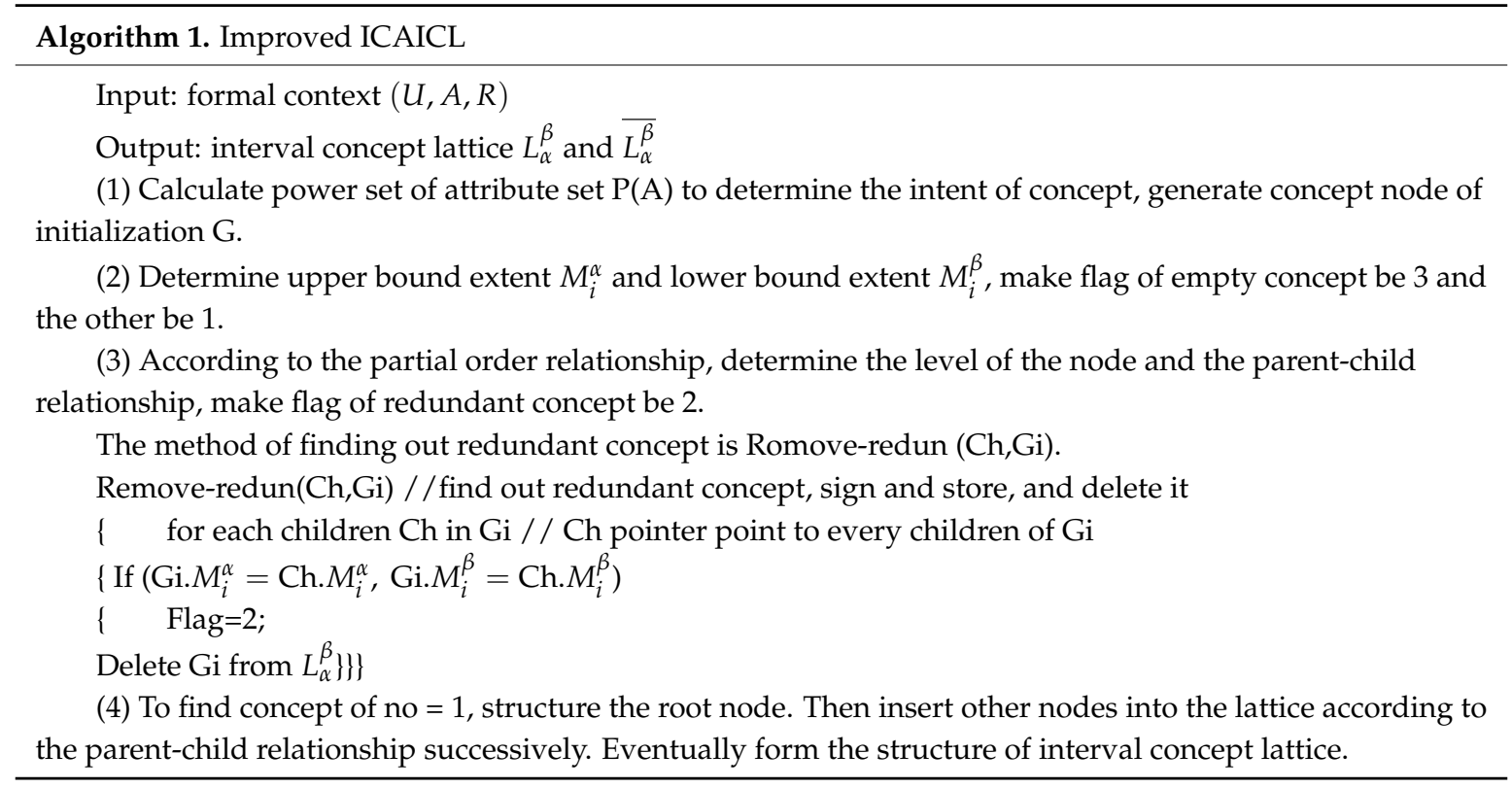

\subsubsection{Dynamic Lateral Uniting Algorithms for Multi-Interval Concept Lattices}

The basic idea of the algorithm is that whenever a new data set is generated, it will be transformed into a formal representation of the interval concept lattice and united with the united lattice structure. In this way, the information of the interval concept lattice can be aggregated again and again, which lays a foundation for further mining association rules.

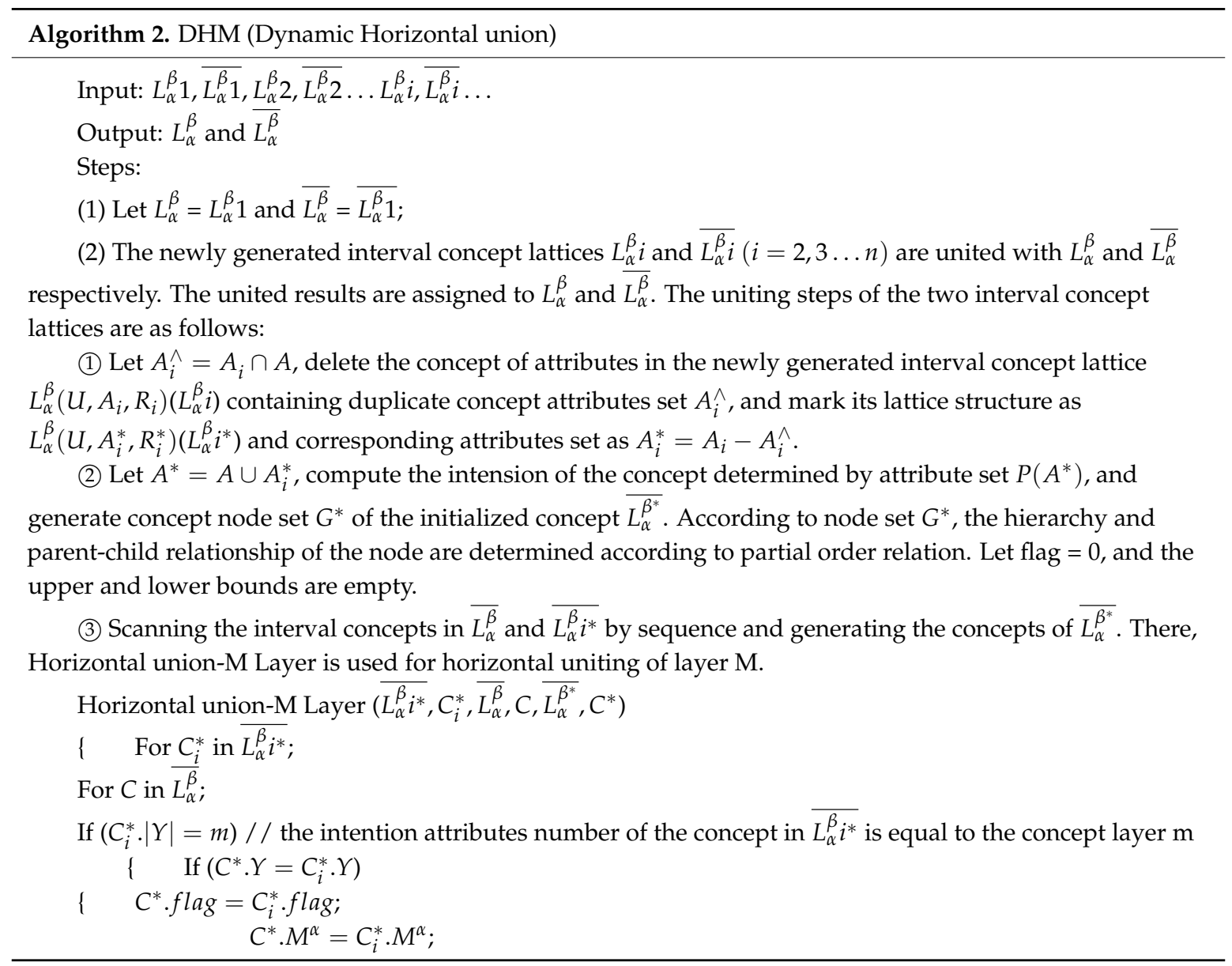


Else end \}

$$
\left.C_{1}^{*} \cdot M^{\beta}=C_{i}^{*} \cdot M^{\beta} ;\right\}
$$

If $(C .|Y|=m) / /$ the intention attributes number of the concept in $\overline{L_{\alpha}^{\beta}}$ is equal to the concept layer $\mathrm{m}$

$$
\left\{\quad \text { If }\left(C^{*} . Y=C . Y\right)\right.
$$

$\left\{\quad C^{*}\right.$. flag $=$ C.flag;

$$
\begin{aligned}
& C^{*} \cdot M^{\alpha}=C \cdot M^{\alpha} ; \\
& \left.C^{*} \cdot M^{\beta}=C \cdot M^{\beta} ;\right\}
\end{aligned}
$$

Else end $\}$ layer $m$

If $\left(C_{i}^{*} .|Y|+C .|Y|=m\right) / /$ The sum of the number of attributes in $\overline{L_{\alpha}^{\beta} i^{*}}$ and $\overline{L_{\alpha}^{\beta}}$ is equal to the concept

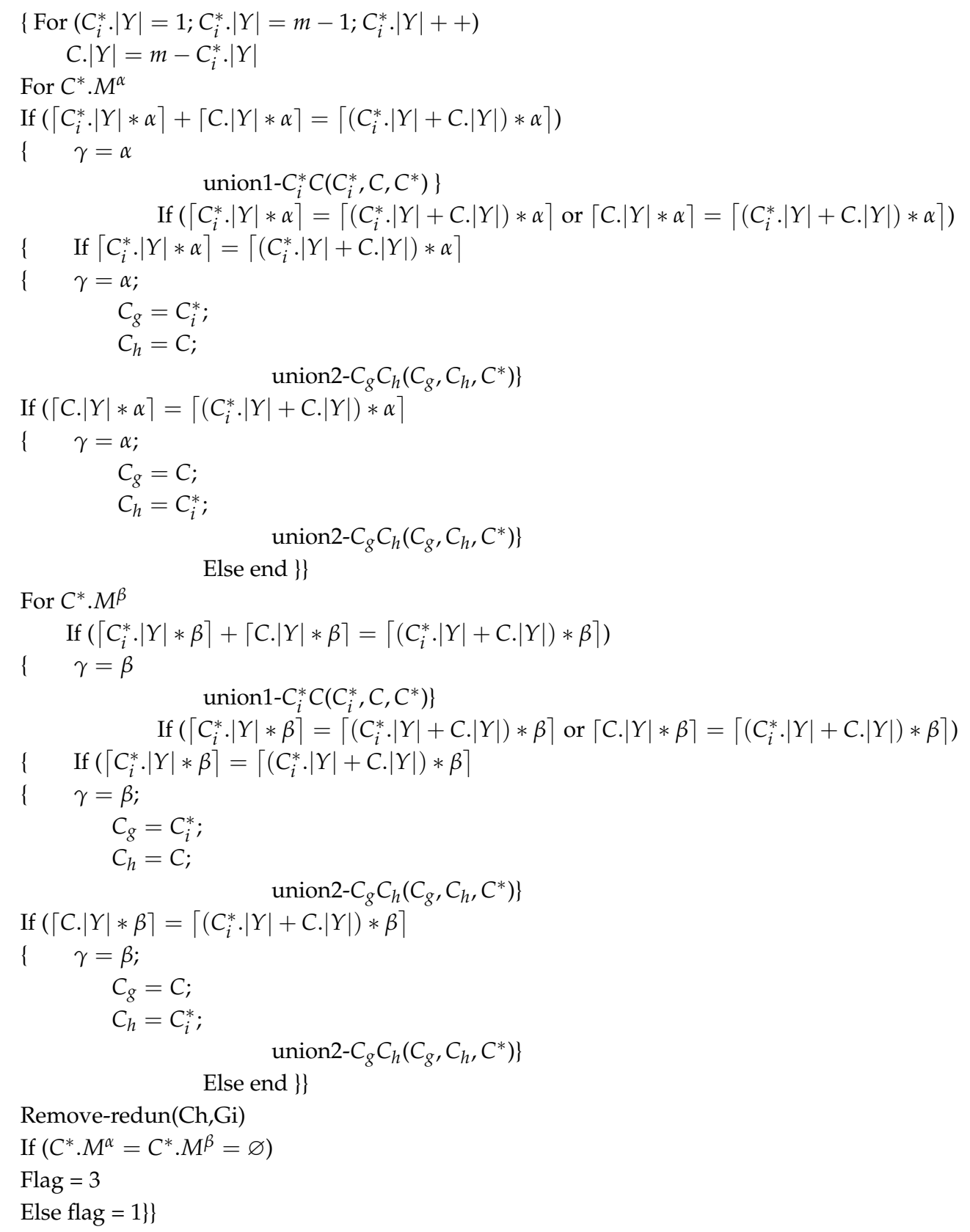

The Horizontal union-M Layer algorithm calls three sub-functions, Remove-redun(Ch,Gi), union1- $C_{i}^{*} C\left(C_{i}^{*}, C, C^{*}\right)$ and union2- $C_{i}^{*} C\left(C_{i}^{*}, C, C^{*}\right)$ respectively. The function of Remove-redun(Ch, Gi) is to find redundant concepts of united interval concept lattices and mark them. The function of 
union1- $C_{i}^{*} C\left(C_{i}^{*}, C, C^{*}\right)$ and union2- $C_{i}^{*} C\left(C_{i}^{*}, C, C^{*}\right)$ are to calculate and test the deleted objects for uniting cases, and to get the concept of interval after uniting.

Assign $L_{\alpha}^{\beta}$ and $\overline{L_{\alpha}^{\beta}}$ to $L_{\alpha}^{\beta^{*}}$ and $\overline{L_{\alpha}^{\beta^{*}}}$ separately. Let $i=i+1$, go to step 2.

The algorithm starts from the interval concept lattice directly, makes full use of the lattice structure of the original interval concept lattice and covers the uniting of all concepts. Therefore, it has completeness and effectiveness. Compared with the method of uniting formal context first and then using ICAICL algorithm to construct the interval concept lattice, this algorithm reduces time complexity and has a value of $O\left(n * n_{i} *\left(n+n_{i}\right)\right)$, which proves the efficiency of the algorithm.

\section{Example Analysis}

Two formal contexts are listed in Tables 1 and $2 . U=\{1,2,3,4,5\}$ is the element set. $A_{1}=\{a, b, c\}$ and $A_{2}=\{c, d, e, f\}$ are attribute sets before and after the change, respectively. Their corresponding lattice structures and union are described as follows.

(1) Set $\alpha=0.6, \beta=0.7$, applying the improved interval concept lattice progressive generation algorithm to generate the primitive lattice structure is shown in Figure $1 \mathrm{a}, \mathrm{b}$.

(2) $A=\{a, b, c, d, e, f\}$. Flag $=0$. The upper and lower boundaries are empty. Initialization of Interval Concept Constitution Generated by Parent-Child Relation is $\overline{L_{\alpha}^{\beta}}$.

(3) $A^{*}=A_{1} \cap A_{2} \neq \varnothing, A^{*}=c$. Deleting all the concept nodes in $\overline{L_{\alpha}^{\beta} 1}$ which contain the attribute $c$. Here, Lattice structure is ${\overline{L_{\alpha}^{\beta}}}^{*}$ and its attributes set is $A_{1}^{*}=A_{1}-A^{*}$.

(4) Scanning ${\overline{L_{\alpha}^{\beta}}}^{*}$ and $\overline{L_{\alpha}^{\beta} 2}$ in sequence, and the interval concept is generated in different cases. $L_{\alpha}^{\beta}$ can be obtained from $\overline{L_{\alpha}^{\beta}}$. The interval concept lattice after horizontal union is shown in Figure 2.

Table 1. The formal context of $L_{\alpha}^{\beta} 1$.

\begin{tabular}{ccccc}
\hline $\mathbf{U}$ & $\mathbf{A}_{\mathbf{1}}$ & $\mathbf{a}$ & $\mathbf{b}$ & $\mathbf{c}$ \\
\hline 1 & 1 & 0 & 1 \\
2 & 0 & 1 & 0 \\
3 & 1 & 1 & 0 \\
4 & 1 & 0 & 0 \\
5 & 1 & 0 & 1 \\
\hline
\end{tabular}

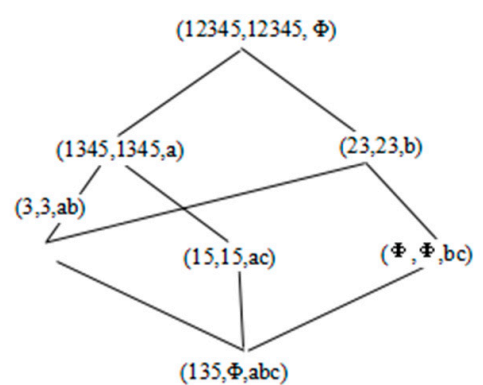

(a)

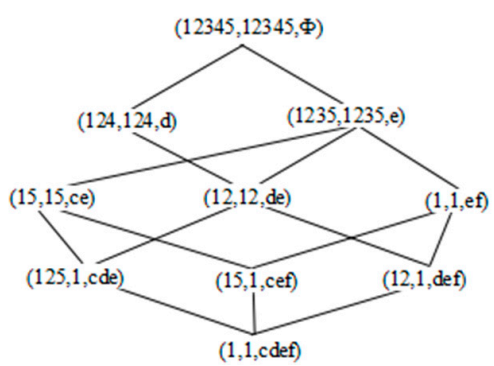

(b)

Figure 1. (a) The lattice structure $L_{\alpha}^{\beta}\left(U, A_{1}, R_{1}\right)$ of $L_{\alpha}^{\beta} 1$; (b) The lattice structure $L_{\alpha}^{\beta}\left(U, A_{2}, R_{2}\right)$ of $L_{\alpha}^{\beta} 2$.

Take Layer 3 as an example, several cases of horizontal uniting of interval concepts are described below. The number of attributes in ${\overline{L_{\alpha}^{\beta}}}^{*}$ and $\overline{L_{\alpha}^{\beta}}$ are 2 and 4 . According to $C_{6}^{3}=C_{4}^{3}+C_{2}^{1} C_{4}^{2}+C_{2}^{2} C_{4}^{1}$, the uniting of the third layer of $\overline{L_{\alpha}^{\beta}}$ can be divided into three cases. 
- Case 1: There are four concepts of the third layer in $\overline{L_{\alpha}^{\beta} 2}$. According to the same principle of intension attributes, the corresponding concepts in $\overline{L_{\alpha}^{\beta}}$ are found, and the upper and lower bounds of the concepts and their flags are assigned to the corresponding interval concepts.

- Case 2: $\operatorname{In}{\overline{L_{\alpha}^{\beta}}}^{*}$, the nodes which have one intension attribute are $(1345,1345, a)$ and $(23,23, b)$, and the nodes in $\overline{L_{\alpha}^{\beta}}$ which have two intension attributes are $(1,1, c d),(15,15, c e),(1,1, c f)$, $(12,12, d e),(1,1, d f)$ and $(1,1, e f)$. Merge them horizontally. Firstly, the uniting case between $(1345,1345, a)$ and $(1,1, c d)$ belongs to $\lceil|c d| * 0.6\rceil=\lceil(|a|+|c d|) * 0.6\rceil$, so $M^{\alpha *}=\{1\}$. Meanwhile, $\{1345\}-\{1\}=\{345\} \neq \varnothing$, bring the elements of $\{345\}$ into $\mid f(x) \cap$ acd $|/| a c d \mid \geq$ 0.6 , determining whether the proportion of intension attributes corresponding to deleted objects satisfies the relationship further, and then add the object into $M^{\alpha *}$ if the relationship is satisfied, otherwise it will be eliminated completely. By analogy, the upper and lower boundary extensions are used to determine the uniting case, and the deletion concept is further verified. Finally, the type of the deletion concept is determined according to the upper and lower boundary extensions after the concept is generated.

- Case 3: Uniting the nodes which have two intension attributes in ${\overline{L_{\alpha}^{\beta}}}^{*}$ and the nodes which have one intension attributes in $\overline{L_{\alpha}^{\beta}}$. The interval concepts obtained from the above three cases together constitute the third layer of $\overline{L_{\alpha}^{\beta}}$.

Table 2. The formal context of $L_{\alpha}^{\beta}$.

\begin{tabular}{cccccc}
\hline $\mathbf{U}$ & $\mathbf{A}_{\mathbf{1}}$ & $\mathbf{c}$ & $\mathbf{d}$ & $\mathbf{e}$ & $\mathbf{f}$ \\
\hline 1 & 1 & 1 & 1 & 1 \\
2 & 0 & 1 & 1 & 0 \\
3 & 0 & 0 & 1 & 0 \\
4 & 0 & 1 & 0 & 0 \\
5 & 1 & 0 & 1 & 0 \\
\hline
\end{tabular}

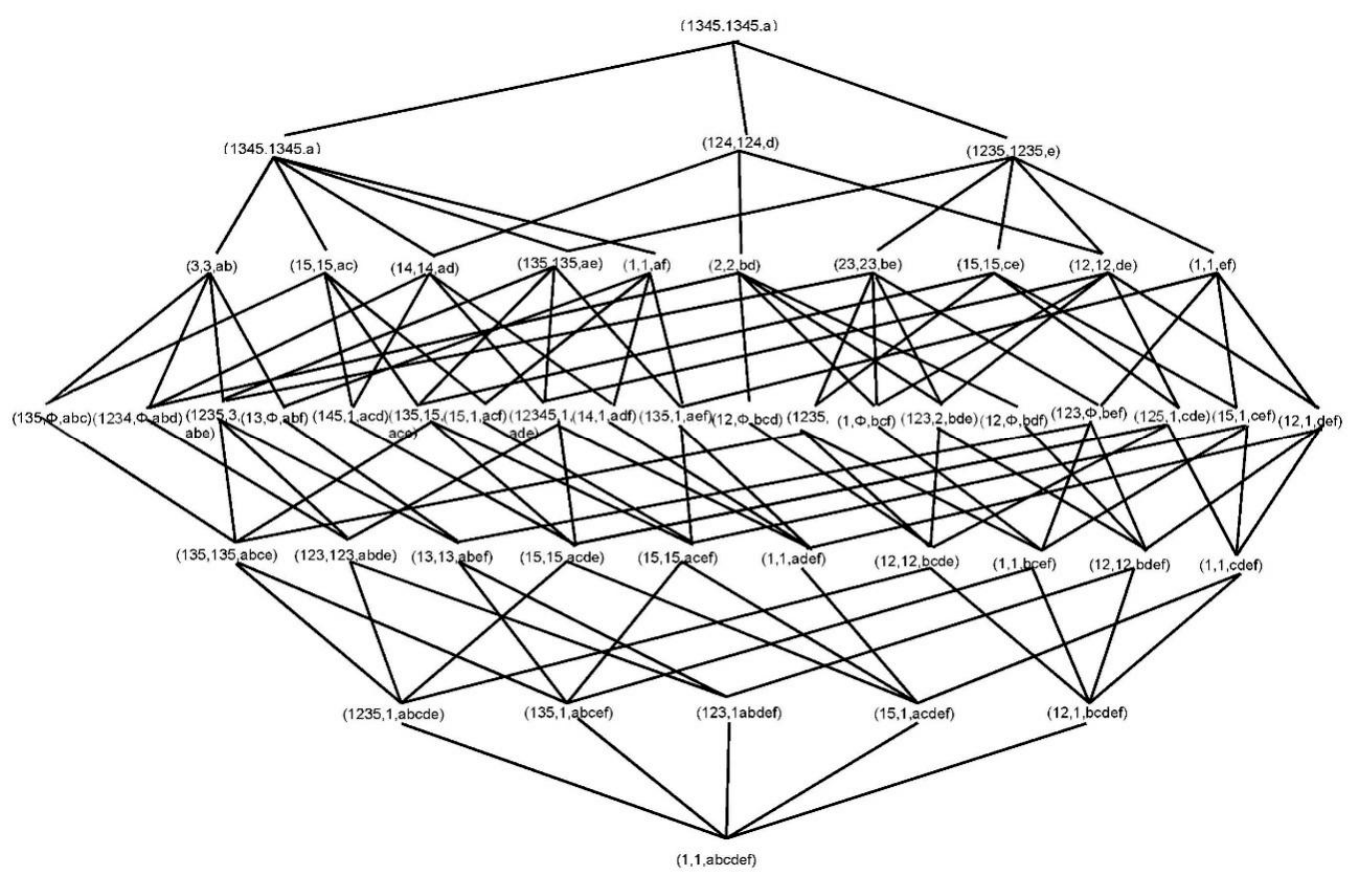

Figure 2. The lattice structure $L_{\alpha}^{\beta}(U, A, R)$ united by $L_{\alpha}^{\beta}\left(U, A_{1}, R_{1}\right)$ and $L_{\alpha}^{\beta}\left(U, A_{2}, R_{2}\right)$ for the Interval parameters $\alpha=0.6$ and $\beta=0.7$. 
Then $\overline{L_{\alpha}^{\beta}}$ can be obtained by horizontal uniting between conceptual nodes. After eliminating the interval concepts of flag equaling 2 and 3 in $\overline{L_{\alpha}^{\beta}}$, it can be transformed into $L_{\alpha}^{\beta}$. See Figure 2.

\section{Conclusions}

When facing real-time data updating in the era of big data, how to deal with data effectively which are generated at any time has become one of the key issues. In this paper, the concept of consistency is introduced as a prerequisite for the uniting of interval concept lattices. Lateral uniting of interval concept lattices can be carried out when the parameter intervals of two interval concept lattices are identical and the object sets are identical. In order to preserve the integrity of the lattice structure, the concepts in lattice structures are divided into three categories: Existential concept, redundant concept and empty concept by improving the progressive generation algorithm of interval concept lattices. The concept of $\overline{L(U, A, R)}$ is introduced at the same time. Lateral uniting of lattice structures is specified to the horizontal uniting of lattice nodes. It can be divided into two situations, that is, the number of connotative attributes of concepts in the original structure equals the number of conceptual layers and the sum of the number of connotative attributes of concepts in the two original structures equals the number of conceptual layers, and the two cases are further refined according to the actual situation, so as to realize the horizontal union of interval concept lattices.

However, in the face of large-scale data, the structure of the interval concept lattice will be greatly expanded, which will lead to high operational complexity. How to develop efficient dynamic uniting software of interval concept lattice, further reduce the complexity of time and space, realize the optimal merging of interval concept lattices, and proposing association rules from them will be the next major research work.

Author Contributions: Y.Y. contributed to the main body of the paper, the improved method of constructing the interval concept lattice, and the principle of dynamic combination. The main models and algorithms were constructed; R.Z. was mainly responsible for the writing of the paper, colleagues performed data processing and graphics drawing; B.L. guided the overall process of the paper, gave the definition of interval concept lattices, and points out the method and feasibility of the combination of interval concept lattices.

Funding: This research was funded by the National Natural Science Foundation of China $(61370168,61472340)$, Natural Science Foundation of Hebei Province (F2016209344).

Acknowledgments: The authors thank Chunying Zhang, Lihong Li and Bin Bai from North China University of Science and Technology for their technical assistance during materials processing and data acquisition.

Conflicts of Interest: The authors declare no conflicts of interest.

\section{References}

1. Wille, R. Restructing lattice theory: An approach based on hierarchies of concepts. In Ordered Sets; Rival, I., Ed.; Reidel: Dordrecht, The Netherlands, 1982; pp. 445-470.

2. Godin, R.; Missaoui, R.; April, A. Experimental compare-eison of navigation in a Galois lattice with conventional information retrieval methods. Int. J. Man-Mach. Stud. 1993, 38, 747-767. [CrossRef]

3. Carpineto, C.; Romano, G. Information retrieval through hybrid navigation of lattice representations. Int. J. Hum.-Comput. Stud. 1996, 45, 553-578. [CrossRef]

4. Cole, R.; Stumme, G. CEM-A conceptual email manager. In Proceedings of the 7th International Conference on Conceptual Structures (ICCS'2000), Darmstadt, Germany, 14-18 August 2000; Springer Verlag: Berlin/Heidelberg, Germany, 2000.

5. Zhang, C.; Liu, B.; Guo, J.; Liu, F.C. Incremental algorithm for building association rule lattice based on attribute linded-list. Comput. Eng. Des. 2005, 26, 320-323.

6. Qiang, Y.; Liu, Z.; Lin, W.; Shi, B.; Li, Y. Research on an Algorithm for Fuzzy Concept Lattice Construction. Comput. Eng. Appl. 2004, 29, 50-53.

7. Fuentes-Gonzlez, B. Construction of the L-fuzzy concept lattice. Fuzzy Sets Syst. 1998, 97, 109-114.

8. Xie, Z.; Liu, Z. Concept Lattice and Association Rule Discovery. J. Comput. Res. Dev. 2000, 37, 1415-1421. 
9. Qiu, G.; Zhu, Z. Acquisitions to Decision Rules and Algorithms to Inferences Based on Crisp-fuzzy Variable Threshold Concept Lattices. Comput. Sci. 2009, 36, 216-218.

10. Xie, Z. Knowledge Discovery Based on Concept Lattice Model. Doctoral Dissertation, Hefei University of Technology, Hefei, China, 2001; pp. 1-20.

11. Zhang, J.; Zhang, S. Weighted Concept Lattice and Incremental Construction. Pattern Recognit. Artif. Intell. 2005, 18, 171-176.

12. Yang, H.; Zhang, J. Rough concept lattice and construction arithmetic. Comput. Eng. Appl. 2007, 43, $172-175$.

13. Liu, B.; Zhang, C. New concept lattice structure-interval concept lattice. Comput. Sci. 2012, 39, $273-277$.

14. Zhai, Y.; Guo, W.; Wang, L. A new association rule classification algorithm using extended concept lattice. J. Liaoning Tech. Univ. (Nat. Sci.) 2015, 34, 1280-1284.

15. Liu, J.; Liu, B.; Chen, H. Rules extraction of fuzzy association rules lattice. J. Liaoning Tech. Univ. (Nat. Sci.) 2013, 32, 852-856.

16. Yao, J.; Yang, S.; Li, X.; Peng, Y. Incremental double sequence algorithm of concept lattice union. Appl. Res. Comput. 2013, 30, 1038-1040.

17. Zhang, L.; Shen, X.; Han, D.; An, G. Vertical union algorithm of concept lattices based on synonymous concept. Comput. Eng. Appl. 2007, 43, 95-98.

18. Li, H. A vertical union algorithm of concept lattices. Nat. Sci. J. Harbin Norm. Univ. 2010, 26, 27-31.

19. Zhang, R.; Zhang, C.; Wang, L.; Liu, B. Vertical union algorithm of interval concept lattices. J. Comput. Appl. 2015, 35, 1217-1221.

20. Zhang, C.; Wang, L. Incremental construction algorithm based on attribute power set for intercal concept lattice $L_{\alpha}^{\beta}$. Appl. Res. Comput. 2014, 31, 731-734.

21. Liu, Z.T.; Shen, X.J.; Wu, Q.; Qiang, Y. Theoretical research on the distributed construction of concept lattices. In Proceedings of the Second International Conference on Machine Learning and Cybernetics, Institute of Electrical and Electronics, Xi'an, China, 2-5 November 2003; pp. 474-479.

22. Zhi, H.; Zhi, D.; Liu, Z. Theory and algorithm of concept lattice union. Acta Electron. Sin. 2010, 38, 455-459.

(C) 2019 by the authors. Licensee MDPI, Basel, Switzerland. This article is an open access article distributed under the terms and conditions of the Creative Commons Attribution (CC BY) license (http:/ / creativecommons.org/licenses/by/4.0/). 